\title{
Toward steering a jet of particles into an x-ray beam with optically induced forces
}

\author{
Niko Eckerskorn, ${ }^{1}$ Richard Bowman, ${ }^{2,3}$ Richard A. Kirian, ${ }^{4,5}$ Salah Awel, ${ }^{4,7}$ Max Wiedorn, ${ }^{4}$ \\ Jochen Küpper, ${ }^{4,6,7}$ Miles J. Padgett, ${ }^{2}$ Henry N. Chapman, ${ }^{4,6,7}$ and Andrei V. Rode ${ }^{1}$ \\ ${ }^{1}$ Laser Physics Centre, Research School of Physics and Engineering, \\ The Australian National University, Canberra ACT 0200 Australia \\ ${ }^{2}$ Department of Physics and Astronomy, University of Glasgow, Glasgow G12 8QQ, UK. \\ ${ }^{3}$ Queens' College, Cambridge Nanophotonics Centre, Cavendish Laboratory, \\ University of Cambridge, CB3 OHE, UK \\ ${ }^{4}$ Center for Free-Electron Laser Science, DESY, 22607 Hamburg, Germany \\ ${ }^{5}$ Department of Physics, Arizona State University, Tempe, AZ, USA \\ ${ }^{6}$ Department of Physics, Universität Hamburg, 22761 Hamburg, Germany \\ ${ }^{7}$ Center for Ultrafast Imaging, Universität Hamburg, 22761 Hamburg, Germany
}

\begin{abstract}
Optical trapping of light-absorbing particles in a gaseous environment is governed by a laser-induced photophoretic force, which can be orders of magnitude stronger than the force of radiation pressure induced by the same light intensity. In spite of many experimental studies, the exact theoretical background underlying the photophoretic force and the prediction of its influence on the particle motion is still in its infancy. Here, we report the results of a quantitative analysis of the photophoretic force and the stiffness of trapping achieved by levitating graphite and carbon-coated glass shells of calibrated sizes in an upright diverging hollow-core vortex beam, which we refer to as an 'optical funnel'. The measurements of forces were conducted in air at various gas pressures in the range from 5 mbar to 2 bar. The results of these measurements lay the foundation for developing a touch-free optical system for precisely positioning sub-micrometer bioparticles at the focal spot of an x-ray free electron laser, which would significantly enhance the efficiency of studying nanoscale morphology of proteins and biomolecules in femtosecond coherent diffractive imaging experiments.
\end{abstract}

Keywords: optical guiding of particles; laser vortex beam; photophoretic force; light pressure

\section{INTRODUCTION}

Optically-induced forces provide a promising route to touch-free guiding of air-borne particles of micrometer and sub-micrometer sizes [1-5]. In general, there are two types of forces responsible for the impact of laser light on light-absorbing particles suspended in gaseous environment. The first is the force of light pressure, due to momentum transfer by photons. The broad field of optical trapping was pioneered by Ashkin [6], who in his first demonstration proposed and developed the basic concept of a counter-propagating gradient-force optical trap. In his experiments he established that the radiation pressure from a continuous-wave laser beam of a few ten $\mathrm{mW}$ could stably hold micrometer-sized dielectric particles in a three-dimensional confinement in air and in vacuum.

The second force is a thermal or photophoretic force $F_{p p}$, which results from the transfer of momentum from gas molecules to the particles and occurs only if the surface of a particle is unevenly heated by an incident light beam. Under anisotropic heating, surrounding gas molecules rebound off the surface with different velocities creating a net force on the particle [7]. This effect was discovered by Ehrenhaft a century ago and termed photophoresis [8]. It depends on the particle size relative to the mean-free path of gas molecules and thus on the gas pressure, the

Optical Trapping and Optical Micromanipulation XII, edited by Kishan Dholakia, Gabriel C. Spalding Proc. of Vol. 9548, 95480H - (c) 2015 SPIE · CCC code: 0277-786X/15/\$18 · doi: 10.1117/12.2191442 
absorption of laser light, and thermal conductivity of the particle [9-11]. The ratio of the gas mean-free path to particle diameter is known as the Knudsen number $\mathrm{Kn}$. The force experienced by an irradiated surface depends on gas pressure $p$ in the following way: $F_{p p}$ is directly proportional to $p$ at extremely low pressures, inversely proportional to $p$ at extremely high pressures, and the plot of $F_{p p}$ over a logarithmic pressure scale is symmetric with respect to the characteristic pressure at which $K n \sim 1$ where $F_{p p}$ attains a maximum as illustrated in Fig.1 [12,13]. Photophoretic forces can be up to three orders of magnitude higher than the radiation pressure at normal atmospheric conditions [3].

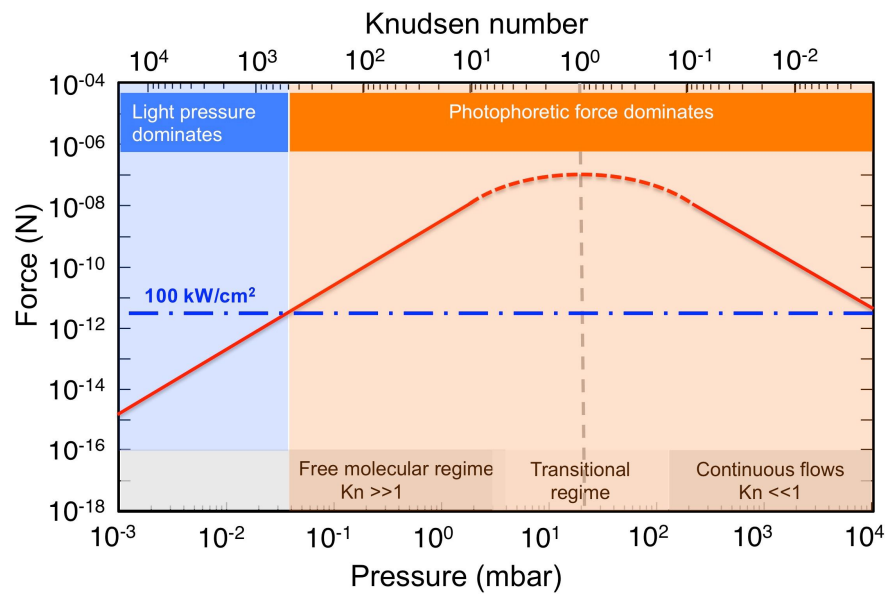

FIG. 1. Illustration of the dependence of photophoretic force (red line) on gas pressure for different regimes of Knudsen number $K n$. The force was calculated for a highly absorbing $1 \mu \mathrm{m}$ diameter particle, for which the $K n \sim 1$ at $\sim 20$ mbar. In the continuum flow regime $(K n<<1)$ the force increases with reduced pressure, followed though the transition area $K n \sim 1$ into the free molecular regime $(K n>>1)$ where the force reduces with reduced pressure. The point where photophoretic force equates with the force of light pressure depends on the illumination intensity. We show, as an example, that at $100 \mathrm{~kW} / \mathrm{cm}^{2}$ the force of light pressure imposed on a $1-\mu \mathrm{m}$ particle is equal to $\sim 3 \mathrm{pN}$, so the light pressure force dominates at pressures below $4 \times 10^{-2} \mathrm{mbar}$.

The value of photophoretic force is characterised by a Knudsen number $K n$. The mean-free path for the gas molecules at normal conditions in air is $\sim 94 \mathrm{~nm}$ and varies inversely with pressure. In the free molecular flow regime, where particles are much smaller than the mean-free path $(K n>>1)$, the photophoretic force can be estimated by kinetic theory in which case reduced pressure results in smaller forces [9-11]. In the continuous regime $(K n<<1)$, the nature of the interaction has a very different mechanism: the particle's motion is due to the thermal creep phenomenon resulting from the particle surface temperature distribution [12-14]. In this regime, the force increases when the pressure is reduced and can be modelled using the equations governing continuous gas media with proper slip flow boundary conditions. Thermal creep is a rarefaction effect, which occurs due to gas microflow along the walls with heat flux and depends on the temperature gradient of the gas along streamlines [9]. It is temperature driven, not pressure driven, as the gas moves in the direction of increasing temperature. The temperature difference, in turn, depends on the particle absorption for the laser light, which is wavelength dependent, and on the thermal conductivity of the particle. In all the above conditions the momentum transfer for a light-absorbing particle is in the direction from hot to cold, thus the particle moves away from regions of high illumination intensity.

A solid theoretical background to predict the photophoretic force in the transitional regime $K n \sim 1$ is still elusive [9,11-13]. Up to date, there is no theory unifying laser trapping by both radiation force and photophoretic force, and there is no united approach that covers the transitional range $K n \sim 1$, of particular interest for trapping and transport of macromolecules [13,14], and indeed the range of interest for this work. For a particle size of $\sim 1 \mu \mathrm{m}, K n \sim 1$ is reached at about 20 mbar (see Fig.1). At lower gas pressures the photophoretic force decreases and at some point becomes lower than the light pressure force. We indicated this pressure of 0.04 mbar in Fig.1 for $1 \mu \mathrm{m}$ particle by taking, somewhat arbitrarily, a $3 \mathrm{pN}$ force from the pressure of light. 
In the $K n<<1$ regime with small particles at atmospheric pressure both radiation pressure and photophoretic forces are linearly dependent on the incoming laser intensity, and both push the light-absorbing particles toward the direction of the beam propagation, and from higher intensity to lower intensity illumination. This is the reason why it is difficult to trap absorbing particles with a Gaussian beam profile [15], where the position at the maximum of beam intensity is unstable and particles are pushed away from the beam axis. An obvious solution to the problem is the use of structured beams with a doughnut intensity profile such as vortex beams, initially proposed by Rubinsztein-Dunlop et al. $[16,17]$ for use in optical tweezers to handle light absorbing particles with tightly focused beams in liquid media. Specifically, the laser vortex beam traps the particle on the axis where the incident intensity is minimal. The particle on the axis is in stable equilibrium because a small transverse perturbation increases the illuminating intensity and forces push it back to the axis. Optical vortices serve not only as a microscopic tool that can hold micrometer-size particles at rest or translate them, but also rotate them about the beam's axis. A large variety of laser beams with complex architecture offered high level of stability and robustness to trapping [18-20].

The first use of optical vortices for trapping and transporting light-absorbing particles in air was demonstrated using counter-propagating coaxial vortex beams [21,22]. Following this work, the influence of the state of vertical or horizontal linear polarization on the axial force, and therefore speed, of horizontally-transported spherical particles was investigated. In this case the particles were slightly shifted off-axis by gravity, which provides an additional degree of freedom in particle manipulation [23]. The effect is due to the breaking of the symmetry in irradiation of a spherical particle and the resulting difference in Fresnel reflectivity and absorption between the $s$ - and $p$-polarized components of the beam. In a vertical geometry with the beam propagation upwards the difference in optically induced force on the levitating particles on the state of polarisation was demonstrated. The particle could be moved between two stable positions upward or downward in the diverging vortex beam by switching the polarization state from azimuthal to radial without changing any other beam parameters [24].

The optical positioning and long-distance transport of absorbing particles in a gaseous environment or in vacuum opens up a previously unattainable domain of optical manipulation suggesting diverse new applications. Several optical schemes based on various beam architectures with minimum intensity field were proposed to trap particles in gaseous environments since the first demonstration. Among those are selective trapping of thousands of particles in a randomly distributed pockets of a speckle-field $[25,26]$, synchronous manipulation of particle ensembles using an optical lattice formed by multiple-beam interference [27], trapping particles in a tapered-ring optical trap diffracted from a circular aperture [28], and in a bottle beam generated using a moiré technique [29]. Optical trapping can also be applied in robust 3D-manipulation of particles using an optical bottle formed inside the focal volume of a lens with a controlled amount of spherical aberration [4,30], or through holographic-based schemes [31]. Besides trapping in 3D, the control of particle trajectory and speed is also important for delivering particles to measurement apparatuses for morphology and composition studies. Such techniques include a diverging quasi-Bessel beam [32] and/or an air-filled photonic crystal fiber [5,33].

The present study is motivated by our recently introduced concept of utilizing photophoretic forces for the purpose of single-particle diffractive imaging at an X-ray free-electron laser (XFEL). XFELs are among the latest generation of $\mathrm{x}$-ray sources and are unique in their ability to produce few-femtosecond-duration pulses of few-mJ pulse energy. Remarkably, XFELs can combine atomic-spatial resolution imaging with femtosecond temporal resolution. Single-particle diffractive imaging is an especially enticing possibility that is enabled by XFELs. This methodology typically consists of delivering a stream of isolated nanoparticles (such as proteins and viruses) across the intense focus of the pulsed, few-femtosecond-duration XFEL beam. Through the aggregation of diffraction patterns from a large number of individual particles, three-dimensional electron density maps can be formed without prior knowledge of the particle structure, and time-resolved studies can be enabled through, for example, an optical pump pulse that precedes the delayed x-ray probe pulse. At present, one of the key bottlenecks in single-particle data collection at XFELs is caused by the small size of the 100-nm diameter x-ray beam in comparison to an aerodynamically focused particle stream of a few tens of micrometers [34]. This mismatch between the particle stream size and $\mathrm{x}$-ray beam size results in low sample delivery efficiency - only about one in $10^{12}$ particles are intercepted in the case of a $50-\mu \mathrm{m}$ particle beam moving at $200 \mathrm{~m} / \mathrm{s}$ across a $100-\mathrm{nm}$ x-ray beam. As a result, lot's of, often precious, sample is wasted and days of data collection are often required in order to obtain only a few hundred or perhaps a thousand high-quality diffraction patterns at an x-ray pulse repetition rate of $120 \mathrm{~Hz}$, whereas > 100,000 patterns are required for atomic-resolution imaging [35]. 
In the context of single-particle imaging, optically induced forces are appealing due to their ability to enable a level of "touch-free" positioning precision that so far has not been achieved through aerodynamic focusing schemes. Our proposed scheme employs a diverging optical vortex beam as an 'optical funnel' to compress and slow down a spray of fast-moving aerosolized particles initially formed by an aerodynamic lens [36]. The aerodynamic lens [36] produces a nearly collimated beam of particles with a beam diameter and speed depending on the size of the particle. Smaller particles tend to move faster (up to about $400 \mathrm{~m} / \mathrm{s}$ for $10 \mathrm{~nm}$ particles) and produce larger beams when compared to bigger particles. Reducing the particle beam diameter and/or particle speed can increase the fraction of $\mathrm{x}$-ray pulses that intercept a particle. For instance, a $1-\mu \mathrm{m}$-particle beam moving at $1 \mathrm{~m} / \mathrm{s}$ would reduce data collection time by a factor of about 10,000 when compared to a typical beam of $50-\mu \mathrm{m}$ diameter and $200 \mathrm{~m} / \mathrm{s}$ speed. The difficulty in predicting the magnitude of optically induced forces is complicated by the fact that the aerosol injector produces particles suspended in a mixture of both the carrier gas and water vapour, which undergoes rapid expansion into a vacuum chamber maintained at $\sim 10^{-1}-10^{-6}$ mbar. While most of the water from the initial mist of particle-laden microdroplets evaporates prior to reaching the vacuum chamber, the exact residual pressure and concentration of water vapour in the area near the injector nozzle is typically unknown and uncontrolled, and the gas density and velocity field at the x-ray interaction point are not well characterized. For this reason, the prediction of the force can, at this point, only be made from model experiments, where the upper level of optical force is limited by a photophoretic force at atmospheric pressure, and the lower limit is due to the radiation pressure in vacuum. Clearly, considerable empirical investigation of the optical force acting on the particles in the diverging doughnutshaped laser beam is required to predict the beam intensity and divergence to effectively confine the injected particles into the few-micrometer size $\mathrm{x}$-ray focus.

As an important step toward achieving our ultimate goal, we calibrate the optical force imposed by the diverging vortex beam by levitating absorbing particles of various micrometer-size graphite and graphite-coated glass shells at a broad range of laser power and air pressure. We recovered the axial force from the known particle mass and the radial force by measuring stiffness of trapping in the transverse plane and the standard deviation of the particle displacement from the equilibrium position at the beam axis due to the Brownian motion in the static levitating conditions. These force measurements will allow us to determine the requirements for a high-precision, touch-free, optical delivery system for pinpoint injection of sub-micrometer particles into a micrometer-size focus of an XFEL.

\section{LEVITATING PARTICLES IN A DIVERGING VERTICAL VORTEX BEAM}

\subsection{Light induced force on a spherical particle}

\subsubsection{Experimental setup}

The particle trap is arranged by a diverging beam above the focal plane where the potential well is formed on the axis with minimum irradiation intensity. The equilibrium position in the axial direction is determined by the counterbalance between the gravitational downward force $F_{m g}=m g$ and the photophoretic and light pressure

forces induced by the beam: $F_{r a d}+F_{p p}=m g$, where $m$ is the mass of the particle and $g$ the acceleration due to gravity. The levitating forces are recovered from the known mass and the size of the particle suspended at a particular equilibrium position in the diverging beam. We note here that both forces, $F_{r a d}$ and $F_{p p}$, acting on the illuminated surface are linearly dependent on laser intensity. In addition to both light-induced forces, buoyancy can potentially play a role in particle levitation, depending on the particle size and mass. For $2 \mu \mathrm{m}$ and $20 \mu \mathrm{m}$ solid carbon particles, the forces exerted by buoyancy at atmospheric pressure are $\sim 5 \times 10^{-17} \mathrm{~N}$ and $\sim 5 \times 10^{-14} \mathrm{~N}$, respectively, whereas the gravitational forces are on the order of $10^{-13} \mathrm{~N}$ and $10^{-10} \mathrm{~N}$, i.e, the former are three orders of magnitude larger. Buoyancy will therefore be neglected in all further considerations. We note that the particles are always denser than a gas by orders of magnitude in case of solid particles and at least by a factor of hundred in case of hollow spheres with a thin shell.

The stiffness of the trap in the transverse plane is determined from thermal motion of the particles observed with a fast CCD camera. The entire setup is contained within a vacuum chamber to conduct the experiments over a broad range of air pressures. 
A linearly polarized Gaussian beam emitted from a continuous wave (CW) laser of wavelength $\lambda=532 \mathrm{~nm}$ (Coherent Verdi V5) is converted into a single-charge vortex beam with a doughnut shaped intensity cross-section using a 16-step radial phase plate (Holo/Or) (Fig.2a). The incident beam with a doughnut mode is directed upward and focused with a 10x microscope plan achromat objective (Mitutoyo Plan APO 10-x) with NA=0.28, focal length $f$ $=20 \mathrm{~mm}$ and working distance of $33.5 \mathrm{~mm}$. The radius of the vortex ring, i.e., of the maximum intensity ring, in the focal spot $w_{1}=w_{0} / \sqrt{2}$ is $\sim 2.2 \mu \mathrm{m}$. Here $w_{0}=3.1 \mu \mathrm{m}$ is the beam waist of a Gaussian beam. Beyond the Rayleigh range of $z_{0}=\pi w_{0}^{2} / \lambda=56 \mu \mathrm{m}$ the beam diverges with a half-angle $\theta=3.1^{\circ}$ so that the radius of the doughnut beam $r_{b}$ changes with distance $z$ from the focal plane as $r_{b}=w_{1}\left[1+\left(z / z_{0}\right)^{2}\right]$ (see Fig.2b).

The displacement of a trapped particle in the transverse plane from the axis induces an asymmetry in the intensity distribution on the surface of that particle (Fig. 2c). The irradiation asymmetry creates a net restoring photophoretic and light-pressure forces, pushing particle back to the axis. For small displacements the restoring force is linear.

The radiation pressure $\Omega_{r a d}=(1+R) I / c$ is caused by the momentum transfer from photons, where $R$ is reflectivity, $I$ is the laser intensity (power/area), and $c$ is the speed of light. The force exerted by the CW laser beam on the particle is $F_{r a d}=\int \Omega_{r a d} d S$, where $S$ is the spherical surface area illuminated by the beam. For example, focusing a power of $10^{-3} \mathrm{~W}$ into an area of $1 \mu \mathrm{m}^{2}$ results in an intensity of $\sim 10^{5} \mathrm{~W} / \mathrm{cm}^{2}$ and yields a radiation pressure force of $F_{\text {rad }} \sim 3 \mathrm{pN}$, as given in Fig. 1 .

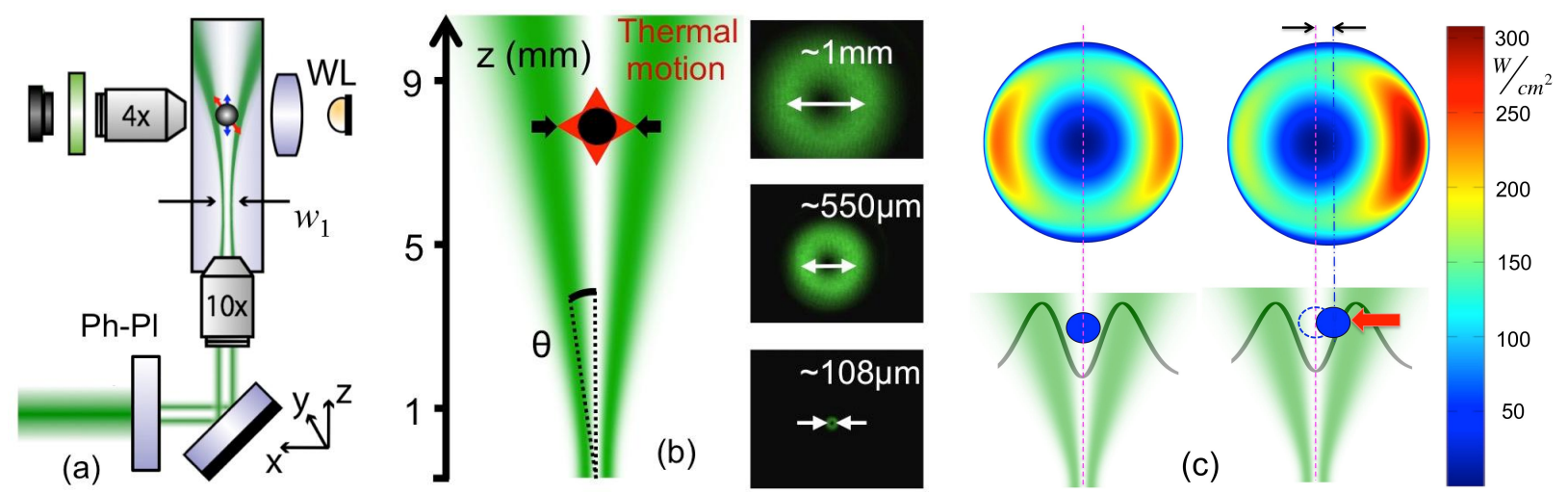

FIG. 2. Levitating particles in a diverging vortex beam. (a) - Experimental setup of 'optical nanoscales'; $\mathrm{Ph}-\mathrm{Pl}$ - is the phase plate to form a vortex beam; 10x - a microscope objective to form the optical funnel; $4 \mathrm{x}$ - microscope objective to track particle motion illuminated by white light (WL); $w_{1}$ - is the vortex beam waist. (b) - The measured profile of the doughnut-shaped vortex beam and the cross sections $2 r_{b}$ at various distances from the focal plane. (c) - Two cases of calculated intensity distribution over a $5 \mu \mathrm{m}$ particle, located $\sim 1 \mathrm{~mm}$ above the focus, and projected on the $\mathrm{y}-\mathrm{z}$ plane; the left one is for a particle on the $\mathrm{z}$-axis and the right is for a particle shifted $1 \mu \mathrm{m}$ away from the z-axis. The polarisation is horizontal, the offset is in the direction of this polarisation. The absorbed laser intensity on the particle surface was calculated for the total beam power of $1.0 \mathrm{~W}$; the particle-to-vortex size ratio is $1: 20$.

\subsubsection{Intensity distribution over a spherical surface}

Both forces $F_{r a d}$ and $F_{p p}$ are governed by irradiation intensity on the particle surface. The intensity distribution across the vortex beam with first topological charge $l=1$ is given by [22]: 


$$
I(r, z)=P_{t o t} \frac{r^{2}}{\pi w_{1}^{4} \xi^{2}} \exp \left(-\frac{r^{2}}{w_{1}^{2} \xi}\right) ; \quad \xi=\left(1+\frac{z^{2} \lambda^{2}}{4 \pi^{2} w_{1}^{4}}\right) ;
$$

where $r$ and $z$ are radial and axial coordinates, $P_{t o t}$ is the total laser power in the beam, $w_{l}$ is the waist of the vortex beam. To create an optical funnel with a controlled beam expansion angle, we focus the beam with a lens with focus $f$. The angle of the cone of the funnel is $\theta^{\prime}=\theta_{0} M$, where $\theta_{0}$ is the Gaussian beam divergence and $M$ is the magnification of the focusing lens (Fig. 2b).

The force acting on a spherical particle with radius $r_{p}$ illuminated by a vortex beam is determined by the axial component of the intensity at each point of the particle surface which can be found using Fresnel equations separately for $s$-and $p$-component [23]:

$$
I_{\text {absorbed }}=I\left[\left|\vec{e}_{s}\right|^{2} A_{s}+\left|\vec{e}_{p}\right|^{2} A_{p}\right] \cos \theta_{i}
$$

where $\vec{e}_{s}$ and $\vec{e}_{p}$ are the $s$ - and $p$-components of the incident laser field on a point on the spherical particle, $\theta_{i}$ the polar angle of incidence, and $A_{s, p}=1-R_{s, p}$ are, correspondingly, the Fresnel absorption and reflectivity for the two components. An example of calculations of the absorbed laser intensity distribution over the sphere positioned at the axis and shifted from the axis is presented in Fig.2 (c) for 1/20 particle-to-vortex radius ratio.

\section{Photophoretic force in continuum flow and in free molecular regimes}

A number of analytical approaches have been developed to predict the dependence of photophoretic force $F_{p p}$ on gas pressure $p$ in both the continuum and free molecular flow regimes. In the free molecular flow regime $(K n>>1)$, the force is the result of gas molecules scattering independently from the particle after having "accommodated" briefly at the surface. The temperature of the gas molecules changes during this process, giving rise to a net force [13]:

$$
F_{p p}^{K n>>1}=\frac{D r_{p}^{2}}{2 \kappa_{p}}\left(\frac{p}{p^{*}}\right) I ;
$$

where $\kappa_{p}$ is thermal conductivity of the particle of radius $r_{p}$, and $p^{*}$ is a characteristic pressure:

$$
p^{*}=\frac{3}{\pi} D \frac{T_{g a s}}{r_{p}} ;
$$

$D$ denotes a constant determined entirely by the state of the gas,

$$
D=\frac{\pi}{2} \sqrt{\frac{\pi}{3} \kappa} \frac{\bar{c} \eta}{T_{g a s}}
$$

where $\kappa$ is a thermal creep coefficient, $0.9<\kappa<1.14$ [13], $\bar{c}$ is the mean speed of the gas molecules, $\bar{c}=\sqrt{8 R T_{\text {gas }} / \pi M_{\text {gas }}}, R$ is the universal gas constant, $M_{\text {gas }}$ is the molar mass, and $\eta$ is the dynamic viscosity of the gas.

In the continuous flow regime $K n<<1$, i.e. at high pressures, tangential temperature gradients in the gas flow cause a tangential flow velocity. This is the so-called thermal creep effect, which arises in a thin gas layer adjacent to the surface of the particle. The force can be expressed as [13]:

$$
F_{p p}^{K n<<1}=\frac{D r_{p}^{2}}{2 \kappa_{p}}\left(\frac{p}{p^{*}}\right)^{-1} I
$$


where $D$ and $p^{*}$ are given by Eqs.(3a,b). In both regimes the photophoretic force is proportional to the illuminating intensity. Taking again the example of a $1 \mu \mathrm{m}$ graphite particle illuminated at atmospheric pressure by a $1 \mathrm{~mW}$ laser beam from a laser pointer focused to $1 \mu \mathrm{m}^{2}$ on the particle surface to produce laser intensity of $10^{5} \mathrm{~W} / \mathrm{cm}^{2}(\bar{c}=493$ $\mathrm{m} / \mathrm{s}$ at $20^{\circ} \mathrm{C} ; \eta=17.8 \mu \mathrm{Pa} \mathrm{s}$ in air), the resulting photophoretic force is $F_{p p} \sim 275 \mathrm{pN}$, or $\sim 75$-times higher than the force of radiation pressure and $\sim 25,000$-times higher than the force due to gravity (see above). We take the thermal conductivity of amorphous carbon $6.3 \mathrm{~W} /(\mathrm{m} \mathrm{K})$, which is quite low when compared to metals, for example. A gold coated surface illuminated by a $532 \mathrm{~nm}$ beam in the same conditions would experience $F_{p p} \sim 1 \mathrm{pN}$, due to high thermal conductivity, $k_{A u}=318 \mathrm{~W} /(\mathrm{m} \mathrm{K})$ and sufficient reflectivity $\sim 0.8$ at this wavelength.

\subsection{Calibration of levitation forces in the optical funnel}

The balance of force in the optical levitating nanoscale trap depends on the total laser power of the beam and on the intersection of the beam by the particle, as well as the environment pressure. The optical force varies along the $\mathrm{z}$-axis for a particle with given radius. Thus, calibration of forces in the trap at a fixed total laser power in the beam was conducted by measuring the equilibrium position of the particle relative to the focal plane and characterised by the ratio of the particle radius to the vortex beam radius $w_{l}$. This ratio was used to determine the illumination of the particle at its equilibrium position. We characterised the optical trap by measuring the equilibrium positions of 64 different particles of various sizes in the beam, and conducted measurements at different cross-sections by varying the laser power for a single particle trapped in the beam. The known mass of the particle allowed us to recover the optically induced force in the axial direction while measuring stiffness of trapping and the root mean square displacement from the axis yielded the force in the transverse cross-section of the beam.

\subsubsection{Mass of glass shells}

To calibrate the forces in the optical funnel we used two types of particles with known masses and recorded the vertical positions of those particles in the funnel for various laser powers and air pressures. We used solid graphite spheres of $2 r_{p}$ in the range $2 \mu \mathrm{m}-12 \mu \mathrm{m}$ (Sigma Aldrich \#484164) for the beam characterisation close to the focal plane, and glass shells (K1, 3MScotchlite) coated with a $30 \mathrm{~nm}-100 \mathrm{~nm}$ graphite layer with diameter ranging from $10 \mu \mathrm{m}$ to $100 \mu \mathrm{m}$ to calibrate forces further away from the focus. The mass of the graphite spheres was evaluated by measuring the size and taking the graphite density $2.26 \mathrm{~g} / \mathrm{cm}^{3}$. In order to determine the mass of glass shells we crushed a number of shells under the optical microscope and measured the wall and the coating layer thickness under field emission scanning electron microscope (FESEM). The result of mass dependence on the shell thickness is presented in Fig. 3. The mass of the shells varies between $1 \mathrm{ng}$ and $100 \mathrm{ng}$, the diameter-to-mass relationship fits the power law $m_{p} \propto\left(2 r_{p}\right)^{2.6 \pm 0.12}$; where $m_{p}$ is in ng and $r_{p}$ is in $\mu \mathrm{m}$. The density of the glass shells varied in size from $\sim 0.05 \mathrm{~g} / \mathrm{cm}^{3}$ to $0.5 \mathrm{~g} / \mathrm{cm}^{3}$, depending on the shell thickness. We note here that while the size of a particle reasonably accurately predicts the mass, thermal properties, which determine the photophoretic force, may vary due to variation in the graphite coating thickness.
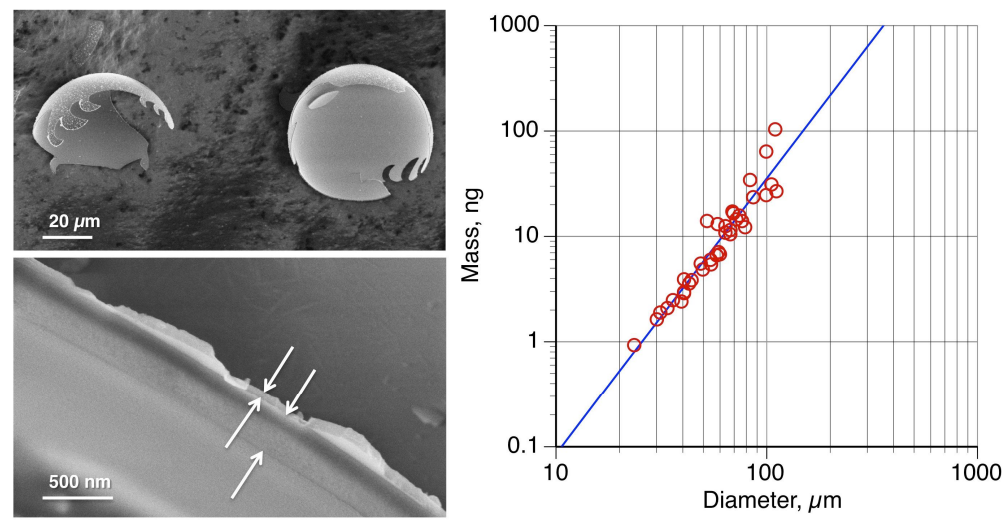

FIG. 3. Measurements of the shell mass. (a) - SEM images of crashed glass shell of $56.8 \mu \mathrm{m}$ in diameter (top) with the glass wall thickness of $350 \mathrm{~nm}$ and graphite coating $65 \mathrm{~nm}$ thick shown in the bottom image. (b) - the 
dependence of the shell mass used in our experiments on the shell diameter. The solid line is the power fit of the shell mass $m_{p}$ to the shell diameter: $m_{p} \propto\left(2 r_{p}\right)^{2.6 \pm 0.12}$.

Particles were inserted into the diverging beam by picking them up with a brush dipped into a vial with samples and repeatedly flicking them from the brush over the top of a glass cylinder that enclosed most of the trapping volume (Fig. 2a). The glass enclosure protects the trap from convective flows in the lab, which otherwise will render the trap extremely unstable when not under vacuum. Once captured within the diverging conical funnel, particles find an equilibrium position as in a potential well and quiver around it due to Brownian motion, fluctuations in beam pointing stability and variation in laser intensity.

\subsubsection{Transverse trapping and axial levitating forces}

To reveal the elastic constant of trapping, or the stiffness of the trap $k(\mathrm{~N} / \mathrm{m})$, we follow well-established methods [19] by tracking a back-illuminated particle's position imaged onto a calibrated CCD at frame rates of $\sim 2$ $\mathrm{kHz}$. The power spectral densities of these position time series show a distinctive dampening of the oscillations with an associated corner or knee frequency, which can be related to stiffness (see Fig. 4). Measurement of the transverse root mean square displacement of the particle from the average position $\langle x-\bar{x}\rangle$ can thus be related to average force: $k\langle x-\bar{x}\rangle=\langle F\rangle$, where $n$ is the number of position measurements [19,37,38]. Additionally, the tracking scheme measures the size of the particle, and therefore axial force can be recovered by knowledge of the size and lateral equilibrium position of the particle and determined from balance against gravity.

In order to accurately track the motion of particles, we passively reduced the beam pointing stability of the optical setup to a standard deviation $<25 \mathrm{~nm}$. As this standard deviation is orders of magnitude below the motion particles undergo while trapped, we can safely neglect the notion of beam pointing instabilities in our further considerations. The laser intensity of our Verdi V5 varies $<1 \%$ of output power, and can be neglected as well. Given that the absorbing nature of our particles causes them to repel from high-intensity regions of the laser beam, a particle within the donut mode, or at least close to the central axis, acts as a harmonic oscillator, as it experiences a restoring force linearly proportional to the displacement from the central axis - the dark core. The power spectral density, which represents the mean square deviation of the particle from the equilibrium in the frequency domain, is used in optical trapping for measuring the trap stiffness.

The particle equilibrium position observed in our experiments was typically in the range of 500 to $1200 \mu \mathrm{m}$ above the focus of the beam, with an average particle size around $10 \mu \mathrm{m}$. The results of the particle motion in the trap are shown in Fig. 4 and Fig. 5. The crossover point, which determines the trap stiffness, is given by the intersection of the frequency-independent oscillation strength due to trapping and a free-diffusion falloff. The transverse stiffness of the optical trap is dictated by both the total power in the trap, and the geometry of the optical trap configuration. A small axial shift of the particle only insignificantly changes absorbed power both along the axis and in the transverse directions, however a transverse offset from the beam axis drastically increases absorbed power (see Fig. 2c). The transverse forces, normalised to the absorbed laser power, are much larger than the normalised axial forces, as shown in Fig. 5.

With the particle in its equilibrium position within the dark core of the optical vortex, the trapping force is strongest at the points of steepest gradient in the illuminating intensity distribution, which are given by the two inner inflection points of the intensity distribution, so that $r_{p} / r_{b}=0.5 \sqrt{5-\sqrt{17}} \cong 0.468, r_{b}$ is the beam radius at the equilibrium position of the particle. Our experiments were conducted with a particle-to-beam ratio much less than 1. As this ratio is decreased, the stiffness of trapping normalised to absorbed power increases (see Fig.5). 

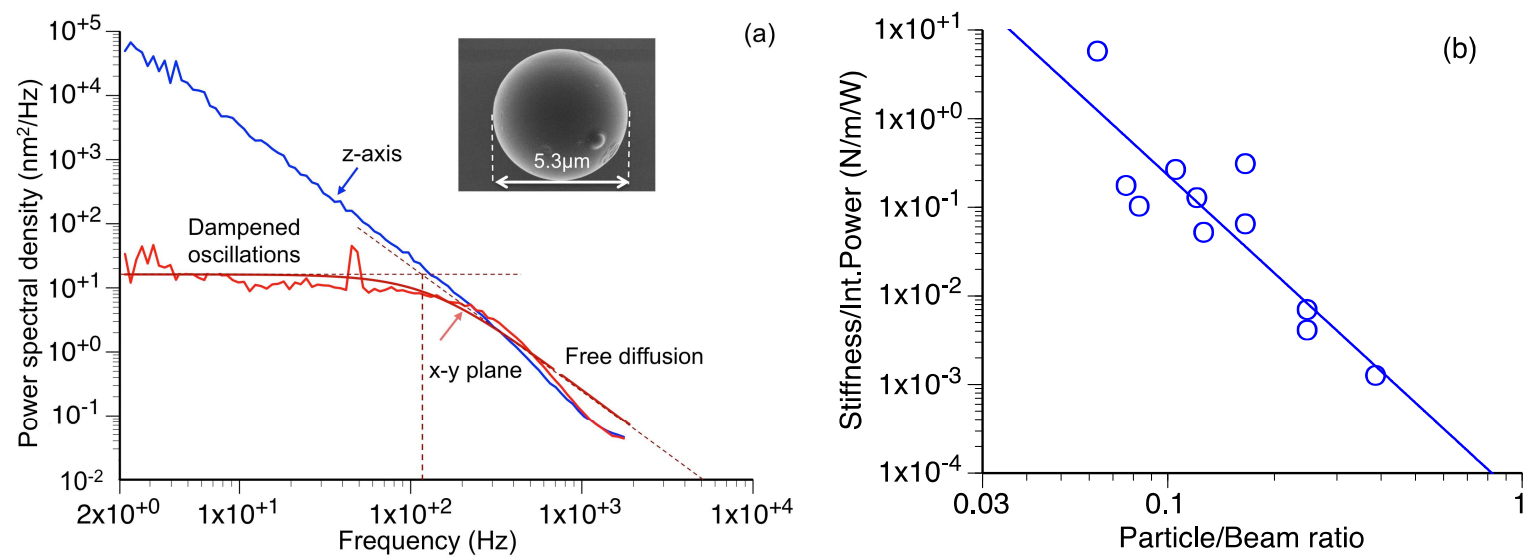

FIG. 4. Analysis of particle movement in the funnel trap at atmospheric pressure. (a) - Example of a power spectrum of a $5.3 \mu \mathrm{m}$ graphite spherical particle motion trapped at atmospheric pressure in an optical potential well of optical funnel in transverse plane (red trace) and in the axial direction (blue trace). The inset shows the SEM image of the particle. The thick solid line is a Lorentzian fit to the transverse position data, while the intersection between the free diffusion and damped oscillations (dashed lines) indicates the corner frequency, which determines the trap stiffness [19]. (b) - The transverse stiffness of the trap normalised to the absorbed laser power recovered from experiments with particles trapped in various positions in the beam.

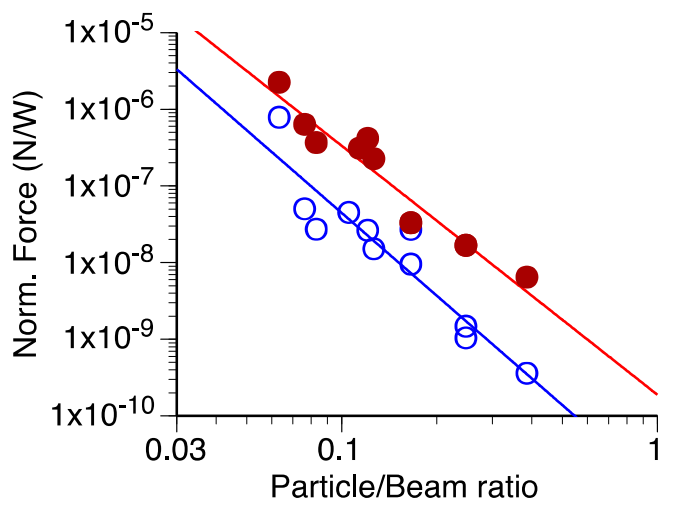

FIG. 5. Transverse trapping force (filled circles) and axial levitating force (hollow circles) normalised to the absorbed laser power recovered from the measurements of trapping stiffness in the transverse plane and by taking the mass of levitating particles along the axis direction. The solid lines are the power fitting to the experimental data.

\subsection{Dependence of photophoretic force on the gas pressure}

Varying the gas pressure changes the photophoretic force and subsequently moves the particle to a new equilibrium position, at which the laser illumination distribution over the particle surface and the particle-to-beam size also changes in the optical funnel configuration. In order to uncover the dependence of photophoretic force on the gas pressure we performed the following experiment: While varying the pressure in the chamber we subsequently varied the laser power to keep the particle in the same position and thus preserved the illuminating geometry. The pressure in the chamber was changed from 2 bar down to atmospheric pressure and further down to $\sim 5$ mbar. As the pressure decreased down to $100 \mathrm{mbar}$, the particles moved to a higher position due to the increased photophoretic force, which consequently required a reduction of the laser power to return particle to the initial position with initial particle-to-beam ratio.

Several graphite-coated glass shells with the mass between $0.2 \mathrm{ng}$ and $0.8 \mathrm{ng}$, the size from $3 \mu \mathrm{m}$ to $11 \mu \mathrm{m}$, and with various particle-to-beam size ratio were used to build the experimental curve in order to check the dependence of the axial force imposed by the particles at different cross-sections of the diverging laser beam - see Fig. 6 . The close coincidence of the required laser power to keep particles of different size in equilibrium positions indicated close thermal properties of particles use in the experiments. 
We observed a linear relationship between the gas pressure above 100 mbar and the trapping power required to levitate a specific particle at a constant position. The experimental data deviate slightly from this linear dependence above atmospheric pressure, which most probably indicates the increase of temperature of the laser irradiated particle and the surrounding gas and the resulting reduced temperature difference across the particle. According to Eq. (3b), the maximum should occur at 29 mbar, which is in agreement with this observation. The transitional region where the photophoretic force goes through its maximum is clearly seen between 10 mbar and 100 mbar. Below 10 mbar the photophoretic force goes down with pressure, which requires higher laser power to keep the particle in a stable position (compare with Fig. 1).

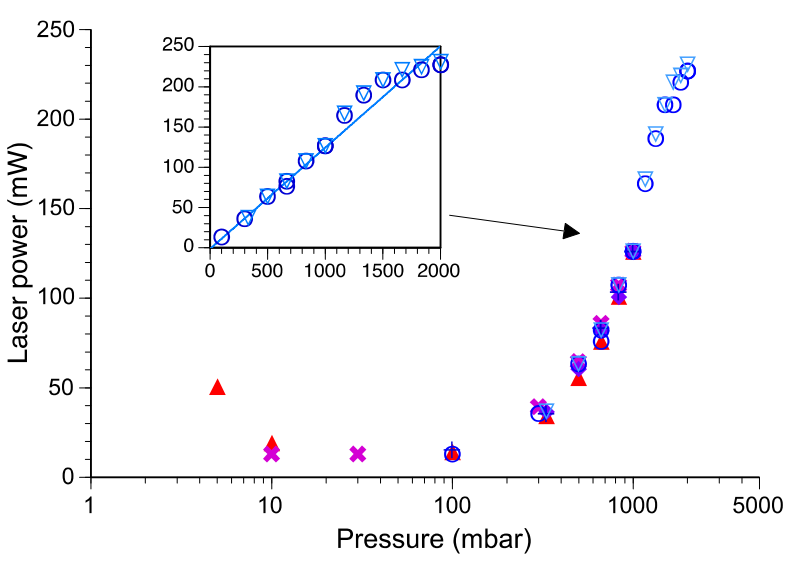

FIG. 6. The relation between the gas pressure and the laser power required to keep the particle in a constant equilibrium position in the diverging beam. The curve was composed of measurements of six particles with different mass and at the following $r_{p} / r_{b}$ ratio in their equilibrium positions: $r_{p} / r_{b}=0.08$ ((1)); 0.11 (1) ; 0.12 $(O) ; 0.12(\nabla) ; 0.13(+) ; 0.22($ 圆). The transitional regime in the $10-100$ mbar range is clearly observed. The characteristic pressure for a $10 \mu \mathrm{m}$ particle in nitrogen $p^{*}=29$ mbar. The linear dependence above $100 \mathrm{mbar}$ is shown in the inset.

\section{CONCLUSIONS AND OUTLOOK}

We have developed a funnel-shaped optical levitation trap and calibrated optical forces in the trap depending on laser intensity and beam geometry. The results of a quantitative analysis of the photophoretic force and the stiffness of trapping achieved by levitating graphite and carbon-coated glass shells of calibrated sizes in an upright diverging hollow-core vortex beam lay the foundation for developing a touch-free optical system for precisely positioning submicrometer bioparticles. We plan to use the optical funnel to enhance the efficiency of sample injection in structural morphology diffraction experiments conducted at free-electron laser facilities. In order to support the operational requirements of free-electron-laser experiments, preferably working in high vacuum or in helium gas at low pressure, our calibration can be extrapolated into the free molecular flow regime. This mapping will allow us to calculate the requirements for focusing a jet of particles as a function of on particle size, position, velocity, and laser power.

\section{ACKNOWLEDGMENTS}

We thank E. G. Gamaly for fruitful discussions. This work has been supported by the excellence cluster "The Hamburg Center for Ultrafast Imaging - Structure, Dynamics and Control of Matter at the Atomic Scale" of the Deutsche Forschungsgemeinschaft (CUI, DFG-EXC1074). This research was partially supported under Australian Research Council's Discovery Projects funding scheme (project number DP110100975). R.K. acknowledges support from the NSF STC award 1231306. J.K. acknowledges support from the European Research Council through the Consolidator Grant 614507-COMOTION.

\section{REFERENCES}

[1] McGloin, D., Burnham, D. R., Summers, M. D., Rudd, D., Dewar, N., Anand, S. "Optical manipulation of airborne particles: techniques and applications", Faraday Discuss. 137, 335-350 (2008). 
[2] Biurnham, D. R., McGloin, D. "Modeling of optical traps for aerosols", Journ. Opt. Soc. Am. B. 28, 2856-2864 (2011).

[3] Shvedov, V. G., Rode, A. V., Izdebskaya, Ya., Desyatnikov, A. S., Krolikowski, W., Kivshar, Yu. "Giant optical manipulation”, Phys. Rev. Lett. 105, 118103 (2010).

[4] Zhang, Z., Cannan, D., Liu, J., Zhang, P., Christodoulides, D. N., Chen, Z. "Observation of trapping and transporting air-borne absorbing particles with a single optical beam", Opt. Express 20, 16212-16217 (2012).

[5] Schmidt, O. A., Garbos, M. K., Euser, T. G., Russell, P. S. J. "Metrology of laser-guided particles in air-filled hollow-core photonic crystal fiber”, Opt. Lett. 37, 91-93 (2012).

[6] Ashkin, A. "Acceleration and trapping of particles by radiation pressure", Phys. Rev. Lett. 24, 156-159 (1970).

[7] Phuoc, T. X. "A comparative study of the photon pressure force, the photophoretic force, and the adhesion von der Waals force", Opt. Commun. 245, 27-35 (2005).

[8] Ehrenhaft, F. “On the physics of millionths of centimeters", Phys. Z. 18, 352-368 (1917).

[9] Davis, E. J., Schweiger, G. "The Airborne Microparticle: Its Physics, Chemistry, Optics, and Transport Phenomena" (Springer-Verlag, Berlin, 2002), pp. 755-810.

[10] Melzer, A. "Laser manipulation of particles in dusty plasmas", Plasma Sources Sci. Technol. 10, 303-310 (2001).

[11] Beresnev, S., Chernyak, V., Fomyagin, G. "Photophoresis of a spherical particle in a rarefied gas", Phys. Fluids. A 5, 2043-2052 (1993).

[12] Weng, H. C., Chen, C-K. "On the importance of thermal creep in natural convective gas microflow with wall heat fluxes", Journ. Phys. D: Appl. Phys. 41, 115501 (2008).

[13] Rohatschek, H. "Semi-empirical model of photophoretic forces for the entire range of pressures", Journal of Aerosol Science 26, 717-734 (1995).

[14] Reed, L. D. “Low Knudsen number photophoresis", Journal of Aerosol Science 8, 123-131 (1977).

[15] Esseling, M., Rose, P., Alpmann, C., Denz, C. "Photophoretic trampoline - interaction of single airborne absorbing droplets with light", Appl. Phys. Lett. 101, 31115 (2012).

[16] He, H., Friese, M. E. J., Heckenberg, N. R., Rubinsztein-Dunlop, H. "Direct Observation of Transfer of Angular Momentum to Absorptive Particles from a Laser Beam with a Phase Singularity", Phys. Rev. Lett. 75, 826-829 (1995).

[17] Rubinsztein-Dunlop, H., Nieminen, T. A., Friese, M. E. J., Heckenberg, N. R. "Optical trapping of absorbing particles", in Advances in Quantum Chemistry: Modern Trends in Atomic Physics edited by P-O. Lowdin (Academic Press, New York, 1998), Vol. 30, pp. 469-492.

[18] Woerdemann, M., Alpmann, C., Esseling, M., Denz, C. "Advanced optical trapping by complex beam shaping", Laser Photonics Rev. 7, 839-854 (2013).

[19] Bowman, R. W., Padgett, M. J. “Optical trapping and binding”, Rep. Prog. Phys. 76, 026401 (2013).

[20] Padgett, M., Bowman, R. “Tweezers with a twist”, Nat. Photonics 5, 343-348 (2011).

[21] Shvedov, V. G., Desyatnikov, A. S., Rode, A. V., Krolikowski, W., Kivshar, Y. S. "Optical guiding of absorbing nanoclusters in air", Optics Express 17, $5743-5757$ (2009).

[22] Desyatnikov, A. S., Shvedov, V. G., Rode, A. V., Krolikowski, W., Kivshar, Y. S. "Photophoretic manipulation of absorbing aerosol particles with vortex beams: theory versus experiment", Optics Express 17, 8201-8211 (2009).

[23] Eckerskorn, N., Zeng, N., Shvedov, V. G., Krolikowski, W., Rode, A. V. "Effect of polarization on air-transport of particle by optical vortex beam", Journal of Optics 14, 055302 (2012). 
[24] Shvedov, V. G., Hnatovsky, C., Eckerskorn, N., Rode, A. V., Krolikowski, W. "Polarisation sensitive photophoresis", Appl. Phys. Lett. 101, 051106 (2012).

[25] Shvedov, V. G., Rode, A. V., Izdebskaya, Y. V., Desyatnikov, A. S., Krolikowski, W., Kivshar, Y. S. "Selective trapping of multiple particles by volume speckle field", Optics Express 18, 3137-3142 (2010).

[26] Shvedov, V. G., Rode, A. V., Izdebskaya, Y. V., Leykam, D., Desyatnikov, A. S., Krolikowski, W., Kivshar, Y. S. "Laser speckle field as a multiple particle trap", Journal of Optics 12, 124003 (2010).

[27] Shvedov, V. G., Hnatovsky, C., Shostka, N., Rode, A. V., Krolikowski, W. "Optical manipulation of particle ensembles in air", Optics Letters 37, 1934-1936 (2012).

[28] Liu, F., Zhang, Z., Fu, S., Wei, Y., Cheng, T., Zhang, Q., Wu, X. "Manipulation of aerosols revolving in tapering optical traps", Opt. Lett. 39, 100-103 (2014).

[29] Zhang, P., Zhang, Z., Prakash, J., Huang, S., Hernandez, D., Salazar, M., Christodoulides, D. N., Chen, Z. "Trapping and transporting aerosols with a single optical bottle beam generated by moiré techniques", Optics Letters 36, 1491-1493 (2011).

[30] Shvedov, V. G., Hnatovsky, C., Rode, A. V., Krolikowski, W. "Robust trapping and manipulation of airborne particles with a bottle beam", Optics Express 19, 17350-17356 (2011).

[31] Čižmár, T., Brzobohatý, O., Dholakia, K., Zemánek, P. “The holographic optical micro-manipulation system based on counter-propagating beams", Laser Phys. Lett. 8, 50 (2011).

[32] Eckerskorn, N., Li, L., Kirian, R. A., Küpper, J., DePonte, D. P., Krolikowski, W., Lee, W. M., Chapman, H. N., Rode, A. V. "Hollow Bessel-like beam as an optical guide for a stream of microscopic particles", Optics Express 21, 30492-30499 (2013).

[33] Schmidt, O. A., Garbos, M. K., Euser, T. G., Russell, P. S. J. "Reconfigurable Optothermal Microparticle Trap in Air-Filled Hollow-Core Photonic Crystal Fiber”, Phys. Rev. Lett. 109, 024502 (2012).

[34] Bogan, M., Boutet, S., Chapman, H. N., et al. "Aerosol Imaging with a Soft X-Ray Free Electron Laser", Aerosol Sci. Technol. 44, 1-6 (2010).

[35] Ayyer, K., Geloni, G., Kocharyan, V., Saldin, E., Serkez, S., Efanov, O., Zagorodnov, I. "Perspectives for imaging single protein molecules with the present design of the European XFEL", Structural Dynamics 2, 041702 (2015).

[36] Chapman, H. N., Fromme, P., Barty, A., et al. "Femtosecond X-ray protein nanocrystallography", Nature (London) 470, 73-77 (2011).

[37] Gibson, G. M., Leach, J., Keen, S., Wright, A. J., Padgett, M. J. "Measuring the accuracy of particle position and force in optical tweezers using high-speed video microscopy", Opt Express 16,14561-70 (2008).

[38] Čižmár, T., Dávila Romero, L. C., Dholakia, K., Andrews, D. L. "Multiple optical trapping and binding: New routs to self-assembly", J. Phys. B: At. Mol. Opt. Phys. 43, 102001 (2010). 